\title{
Bubbles in a minority game setting with real financial data.
}

\author{
Frédéric D.R. Bonnet ${ }^{a, b}$, Andrew Allison ${ }^{a, b}$ and Derek Abbott ${ }^{a, b}$ \\ ${ }^{a}$ Department of Electrical and Electronic Engineering, The University of Adelaide, SA 5005, \\ Australia. \\ ${ }^{b}$ Centre for Biomedical Engineering, The University of Adelaide, SA 5005, Australia.
}

\begin{abstract}
It is a well observed fact that markets follow both positive and/or negative trends, crashes and bubble effects. In general a strong positive trend is followed by a crash - a famous example of these effects was seen in the recent crash on the NASDAQ (april 2000) and prior to the crash in the Hong Kong market, which was associated with the Asian crisis in the early 1994. In this paper we use real market data coupled into a minority game with different payoff functions to study the dynamics and the location of financial bubbles.
\end{abstract}

Keywords: Minority game, dollar game, payoff function, real market data, financial markets, financial bubbles.

\section{INTRODUCTION}

One of the biggest problems with models used in economics and finance is that the relevant features of the market dynamics are sometimes buried under so many parameters that a systematic understanding is almost impossible. This is mainly because the market mechanisms are intrinsically non-linear and complex, which means small variations in any of the parameters could lead to dramatic changes - thus making it difficult to track cause and effect.

To get around this problem physicists usually proceed in constructing models that start from the simplest model, capturing the essential features in question and progressively adding complexities to it. A famous example of this is the Ising model that tries to describe the magnetization in materials.

It is in this spirit that led most agent-based model creators to develop the concept of the Minority Game, which was originally defined by Challet and Zhang. ${ }^{1}$ This model aims at creating a simple but yet rich platform for exploring various phenomena arising from financial markets.

\section{THE MINORITY GAME}

The general idea of the minority game is as follows: at any given time the agents have two choices for example buying or selling, they take their decisions simultaneously without any communication between them, and those who happen to be in minority win. In this context it is not in the interest of any agent to behave in the same way as the rest of the agents.

\subsection{The model}

The dynamics of the Minority Game (MG) is defined in terms of the dynamical variables $U_{s, i}(t)$ in discrete time $t \in \mathcal{N}^{+}$. These are the scores that each agent $i=\{1, . ., N\}$ attaches to each of the possible available choices $s=\{1, \ldots, S\}$. Each agent takes a decision $s_{i}(t)$ with a given probability.

Further author information: (Send correspondence to F.D.R. Bonnet.)

F.D.R. Bonnet.: E-mail: fbonnet@eleceng.adelaide.edu.au, Telephone: +61 8-8-303-6296

A. Allison: E-mail: aallison@eleceng.adelaide.edu.au, Telephone: +61-8-8-303-5283

D. Abbott.: E-mail: dabbott@eleceng.adelaide.edu.au, Telephone: +61-8-8-303-5748

Complex Systems, edited by Axel Bender, Proc. of SPIE

Vol. 6039, 60390C, $(2005) \cdot 0277-786 X / 05 / \$ 15 \cdot$ doi: $10.1117 / 12.673126$

Proc. of SPIE Vol. 6039 60390C-1 


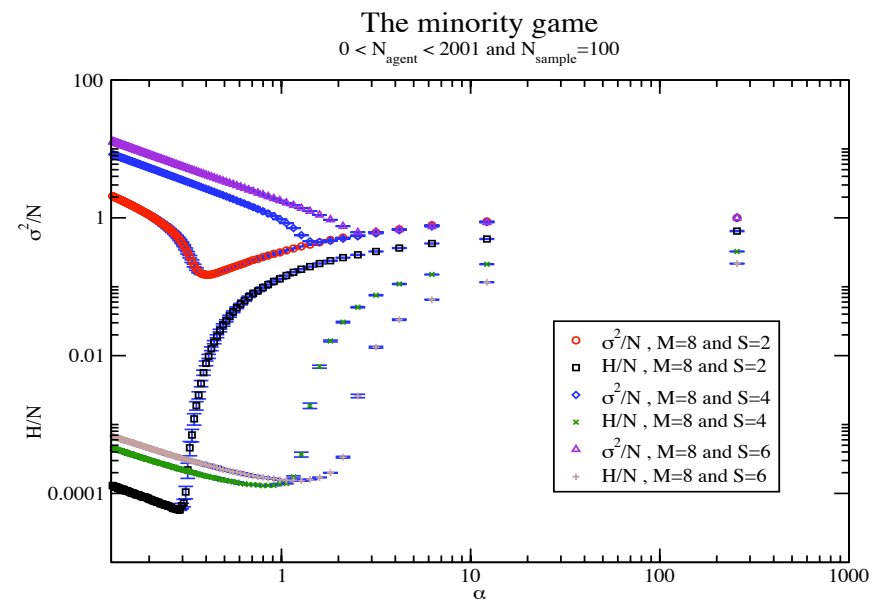

Figure 1. The graph of the global efficiency $\sigma^{2} / N$ and the predictibility $H / N$ versus the critical parameter $\alpha=2^{M} / N$ for a sequence of number of agents varying from 1 to 2001 when $M=8$ and $S=2,4$ and 6 , in each simulation with $(N)_{i}$ number of agents it has been ensemble averaged over 100 samples $\left(N_{\text {sample }}=100\right)$.

The public information variable $\mu(t)$ is given to all agents; it belongs to the set of integers $(1, . ., P)$ and can either be the binary encoding of the last $M$ winning choices ${ }^{1}$ or drawn randomly from a uniform distribution. ${ }^{2}$

The action $a_{s_{i}(t), i}^{\mu(t)}$ of each agent depends on his/her choices $s_{i}(t)$ and on $\mu(t)$. The coefficients $a_{s_{i}, i}^{\mu}$ are either +1 or -1 , and play the role of quenched disorder. These are randomly drawn with probability of a $1 / 2$ for each $i, s$ and $\mu$. They can also be thought of as agents buying (when +1 ) or selling (when -1$)$ an asset. On the basis of the outcome, the action functional, $A(t)=\sum_{i=1}^{N} a_{s_{i}(t), i}^{\mu(t)}$, is used to update each agent score using $U_{s, i}(t+1)=U_{s, i}(t)-a_{s_{i}(t), i}^{\mu(t)} \frac{A(t)}{P}$. Where $P=2^{M}$ is the total number of choices.

Similar results may be obtained when one considers the case when there is a nonlinear dependence on $A(t)$ i.e. with the dynamics $U_{s, i}(t+1)=U_{s, i}(t)-a_{s_{i}(t), i}^{\mu(t)} \operatorname{sgn}[\mathrm{A}(\mathrm{t})]$, where sgn is the usual sign function taking \pm 1 when $A(t)>0$ or $A(t)<0$ respectively. This leads to qualitatively similar results. A more lengthy discussion may be found elsewhere. ${ }^{3-6}$

The source of randomness is in the choice of $\mu(t)$ and by $s_{i}(t)$. These are fast fluctuating degrees of freedom. As a consequence $U_{s, i}(t)$ is also fast fluctuating and hence the probability with which the agents choose $s_{i}(t)$ are subject to stochastic fluctuations.

The key parameter is the ratio $\alpha=P / N$ and the two relevant quantities are

$$
\sigma^{2}=\left\langle A^{2}(t)\right\rangle \text { and, } H=\frac{1}{P} \sum_{\mu=1}^{P}\langle A \mid \mu\rangle^{2},
$$

which measure respectively, (i) the fluctuations of attendance $A(t)$ (i.e. the smaller $\sigma^{2}$ is, the larger a typical minority group is, in other words $\sigma^{2}$ is a reciprocal of the global efficiency of the system) and (ii) the predictability. ${ }^{1}$ Here $\langle\ldots\rangle$ denotes the temporal average.

In Fig. 1, we show the graph of the global efficiency $\sigma^{2} / N$ and the predictibility $H / N$ versus the critical parameter $\alpha=2^{M} / N$ for a sequence of number of agents varying from 1 to 2001 when $M=8$ and $S=2,4$ 
and 6 , in each simulation with $(N)_{i}$ number of agents that has been ensemble averaged over 100 samples $\left(N_{\text {sample }}=100\right)$.

One can observe three different regions in this graph. The first one is found when $\alpha$ is small. In that region fluctuations rapidly increase beyond the level of random agents and the game enters what has been called the crowded region, since it is reached by keeping $M$ constant with $N$ increasing. In other words, agents display herding behaviour and produce non-Gaussian fluctuations $\sigma^{2} \sim N^{2}$, at intermediate $\alpha$, as $N$ decreases that is, when the game enters into a regime where agents manage to coordinate to reduce fluctuations. In other words that is when best coordination is achieved. When $\alpha$ is large, which means that $N$ is small, then the outcome is more or less random. That is, coordination slowly disapears and the variance of the outcome tends to the value that would be produced by agents taking random decisions.

Also the predictability, $H / N$, is shown in Fig. 1. From the graph we can see that the system undergoes a phase transition. Another thing that is worth noting is that the transition point moves according to the minimum shown in the global efficiency graph, Fig. 1, when the number of choices, $S$, is changed.

\subsection{The dollar game}

The minority game is a repeated game where agents, $N$ of them, have to choose one out of two possible alternatives at each step. Each agent, $i$, has a memory of the past. At each time step $t$ every agent decides whether to buy or sell an asset. The agent takes an action $a_{i}(t)= \pm 1$ where 1 is when buying an asset as opposed to -1 when selling. The excess demand $A(t)$ at time $t$ is then given by $A(t)=\sum_{i=1}^{N} a_{s_{i}(t), i}^{\mu(t)}$. The payoff of agent $i$ in the Minority Game is given $g_{i}(t)=-a_{s_{i}(t), i}^{\mu(t)} A(t)$. In order to model financial markets, some authors ${ }^{7,8}$ have used the following definition for the return $r(t)$ using the price time series $P(t)$, that is $r(t) \equiv \ln [P(t)]-\ln [P(t-1)]=\frac{A(t)}{\lambda}$, which means that price time series is defined by,

$$
P(t)=P(t-1) \exp \left[\frac{A(t)}{\lambda}\right] .
$$

Here the liquidity $\lambda$ is proportional to the number of agents $N$. In the minority game the agents predicts the price movements only over the next time step. However, Andersen and Sornette ${ }^{9}$ have shown that in order to know when the price reaches its next local extremum and with optimized gain, the agents need to estimate the price movement over the next two time steps ahead $(t$ and $t+1)$ and they therefore have postulated the correct payoff function to be given by

$$
g_{i}^{\$}(t+1)=a_{i}(t) A(t+1) .
$$

This modification of the minority game is what is called the dollar game (\$-Game).

\section{THE PRICE FUNCTION IN THE MINORITY GAME WITH REAL DATA}

Here we will use the historical price time series of the Nasdaq over a period of about twenty years, that is from October 1984 to late September 2005. During that time we can clearly see the bubble effect of the technological sector from the mid eighties until the bubble burst in the early 2000. The large growth was then followed by a big crash where billions of dollars have been wiped out off the market.

We associate the price movement of the real data with either +1 or -1 in the variable $b_{s_{i}(t), i}^{\mu(t)}$. If the price goes down then this variable takes the value of -1 else +1 . This information is then used to update the value of the history $\mu(t)$, i.e. the history update is implimeneted via $\mu(t+1)=[2 \mu(t)+\operatorname{sgn}[\mathrm{A}(\mathrm{t})] / 2] \bmod \mathrm{P}$.

We also introduce an extra parameter that looks over a certain time in the past, we call it $T$. It can be understood as a window parameter of a given length. 




Figure 2. The graph of the price function as a time series for the $\$$-Game payoff versus MG payoff as a function of time $t$ on a linear scale, $1 \leq t \leq 5283$. This is compared to the real data from the Nasdaq over the period of $11 / 09 / 84$ to the $19 / 09 / 05$, showing clearly the signs of a bubble over the time. Here the number of agents $N=41$ and each agent have $S=2$ strategies to choose from with a memory of $8, M=8$ and with a window size of $T=100$.

Using this window parameter we can compare the dynamics of both games. In Fig. 2 we show the graph of the price function as a time series for the $\$$-Game payoff versus MG payoff in the minority game as a function of time $t$ on a linear scale, $1 \leq t \leq 5283$. This is compared to the real data from the Nasdaq over the period of 11/09/84 to the 19/09/05, showing clearly the signs of a bubble over the time. Here the number of agents $N=41$ and each agent have $S=2$ choices to choose from with a memory of $8, M=8$ and with a window size of $T=100$.

Ignoring the scaling differences between the games and the real data-something that will be studied in future - we can see that in this figure the $\$$-game and the real data follow very similar trajectories as opposed to the minority game, which does not even display the existence of a bubble. So in this figure we can see that the $\$$-game is much more sensitive to the bubble effect, showing clear evidence of peaks and troughs displayed in the real data. There is also clear evidence that there is a scaling problem. This comes from the fact that the liquidity was approximated to be $\lambda \sim N$. However, the liquidity is usually affected, as the market depth is. The market is not constant right through and should be taken as a time series.

As a final test we turn off the dynamics of both games by setting the score updates to 0 , i.e. $U_{s, i}(t+1)=$ $U_{s, i}(t)=0$, so that the scores do not get updated, and see how the game performs on real data, namely on the Nasdaq. This is shown in Fig. 3.

In Fig. 3, we can see that the dynamics of the MG with the \$-Game clearly follow the market data, however this has little benefit because since the scores are not being updated reducing the choices to a coin flip. This makes the MG just follow the path of the real data without really picking up the real dynamics of the market. This is something that will need to be explored further in later works.

\section{CONCLUSION}

In this paper we have shown that it may be possible to use agent-based models such as the minority game for studying bubbles and crashes. This paper does not give a prescription on how to detect bubbles, this will be 


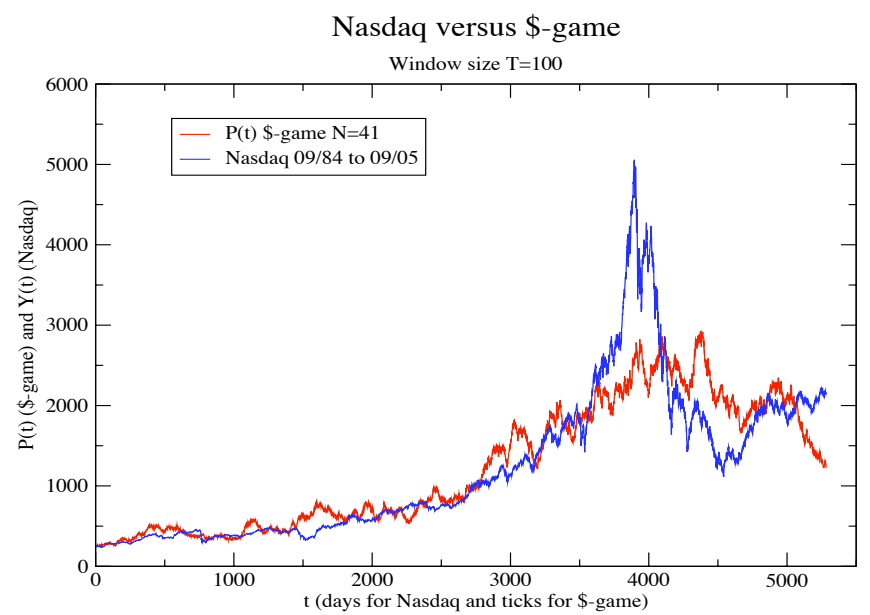

Figure 3. The graph of the time series for the Nasdaq versus the $\$$-Game price function as a function of time $t$, $1 \leq t \leq 5283$ over the period of $11 / 09 / 84$ to the $19 / 09 / 05$, showing clearly the signs of a bubble over the time. Here the number of choices is $S=2$ and the memory is $M=8$.

subject of future work, however we can see clear evidence that the $\$$-Game reproduces the dynamics of the data observed in real markets. We also saw that the minority game payoff does not quite pick up the effects observed in the real market data and that when we change the payoff function to the one used in the dollar game the agent model appears to follow the real data more satisfactorily.

This work is still ongoing and further results will be presented in the near future.

\section{ACKNOWLEDGMENT}

We gratefully acknowledge funding from the Australian Research Council (ARC). Useful discussions with J.V. Andersen and D. Sornette are also very gratefully acknowledged.

\section{REFERENCES}

1. D. Challet and Y.-C. Zhang, "Emergence of cooperation and organization in an evolutionary game," Physica A 246, p. 407, 1997.

2. A. Cavagna, "Irrelevance of memory in the minority game," Phys. Rev. E 59, p. R3783, 1999.

3. M. Marsili, D. Challet, and R. Zecchina, "Exact solution of a modified El Farol's bar problem: Efficiency and the role of market impact," Physica A cond-mat/9908480 280, p. 522, 2000.

4. Y.-C. Zhang Europhys. News 29, p. 51, 1998.

5. R. Savit, R. Manuca, and R. Riolo, "Adaptive competition, market efficiency, and phase transitions," Phys. Rev. Lett. adap-org/9712006 82, p. 2203, 1999.

6. D. Challet, A. Chessa, M. Marsili, and Y.-C. Zhang, "From minority games to real markets," Quant. Fin. adap-org/0011042 212, p. 1, 2001.

7. J.-P. Bouchaud and R. Cont, "A Langevin approach to stock market fluctuations and crashes," Eur. Phys. J. B 6, p. 543, 1998.

8. J. D. Farmer, "Market force, ecology and evolution," adap-org/9812005, 1998.

9. J. V. Andersen and D. Sornette, "The \$-game," Eur. Phys. J. B 31, pp. 141-145, 2003.

Proc. of SPIE Vol. 6039 60390C-5 